\title{
Disorder mapping in VCSELs using frequency-selective feedback
}

\author{
T. Ackemann, ${ }^{1, *}$ N. Radwell, ${ }^{1,2}$ Y. Noblet, ${ }^{1}$ and R. Jäger ${ }^{3}$ \\ ${ }^{1}$ SUPA and Dept. of Physics, University of Strathclyde, Glasgow G4 ONG, UK \\ ${ }^{2}$ Now at SUPA and Dept. of Physics and Astronomy, University of Glasgow, Glasgow G12 8QQ, UK \\ ${ }^{3}$ ULM Photonics GmbH, Lise-Meitner-Str. 13, 89081 Ulm, Germany \\ *Corresponding author: thorsten.ackemann@strath.ac.uk
}

Received November 16, 2011; revised February 3, 2012; accepted February 13, 2012; posted February 14, 2012 (Doc. ID 158130); published March 14, 2012

\begin{abstract}
We report on a simple method with a high spectral and spatial resolution for mapping variations in the cavity resonance of a plano-planar broad-area laser based on frequency-selective feedback. The demonstration experiment uses a vertical-cavity surface-emitting-laser (VCSEL), in which growth induced inhomogeneities are of particular importance. It relies only on a standalone laser with a narrow-bandwidth passive filter avoiding the need for an expensive tunable laser or high-resolution spectrometer. ( 2012 Optical Society of America

OCIS codes: $140.5960,140.7260,140.4780,120.4290,190.4420$
\end{abstract}

A high uniformity of the resonance condition of laser cavities - and hence of the emission wavelength — is very important not only for laser arrays and broad-area lasers but also for the yield in gas sensing and dense wavelength multiplexing communication applications. These considerations are particularly important for semiconductor lasers due to their plano-planar cavities, but are also relevant for other monolithic laser designs with flat end mirrors. In particular, vertical-cavity surface-emitting lasers (VCSELs) require on the order of 100 layers to grow and the cavity resonance is defined by the interference conditions between all these epitaxial grown layers. With modern semiconductor growth technologies like molecular beam epitaxy (MBE) and metalorganic chemical vapor deposition (MOCVD), systematic large scale gradients and inhomogeneities can be reduced to below $\pm 0.2 \%$ over a 3 inch wafer [1] , but the question of growth irregularities on small scales remains and has potentially harmful implications for applications. For example, a typical fingerprint of disorder, coherent backscattering, or weak localization is found in semiconductor microcavities [2]. Inhomogeneities limit the coherence of VCSELarrays [3] and hence their usefulness as high-power sources or for beam steering. One other repeated observation is that broad-area VCSELs tuned to emit onaxis [4] display irregularly shaped filamentary spots instead of a fundamental mode filling the aperture. Inhomogeneities are also very important in the strong coupling regime [ㅁ]. Cavity solitons [ㅁ] are self-localized bistable waves in a nonlinear cavity and should be able to exist anywhere in the plane of a homogenous planoplanar cavity, but will couple to any perturbation of this translational symmetry. Hence the solitons are trapped in local minima of a "potential" landscape, which is created by the growth imperfections [6-10].

It was suggested that this sensitivity of soliton position to growth imperfections can be used to probe the underlying disorder [9] instead of measuring spatially resolved reflection spectra of the microcavity with a tunable, narrow-linewidth laser $[5,11]$. In [9] a mapping is demonstrated by looking at preferred locations of cavity solitons in a VCSEL with external injection operated below threshold, i.e., as an amplifier, but a quantitative in- terpretation is not straightforward, and the experiment is mainly sensitive to identifying the "contour line" where the cavity resonance has a certain detuning (corresponding to the transition between the existence of solitons and extended states in a homogeneous system) to the frequency of the injected field, though this works with a high resolution. In addition, one stills needs a tunable laser of high spatial and temporal coherence.

We propose a scheme using a VCSEL with frequencyselective feedback that can operate stand alone with any VCSEL or optically pumped VECSEL gain chip, i.e., without the need for a tunable laser. It allows quantitative analysis of the fluctuations in the cavity resonance. Though it occurs in a system that shows cavity solitons at threshold $[\underline{8}, 10]$, it does not use this property, but only the sensitivity of the laser losses being in or out of resonance with a narrow band filter in an external cavity.

The experimental setup is described in detail in [10]. A VCSEL is coupled to a frequency-selective element via a self-imaging external cavity. This provides a 1:1 mapping of each point of the VCSEL onto itself on subsequent round trips, thus providing a local probe needed for mapping. The imaging optics of the cavity consists of an aspheric lens with focal length of $8 \mathrm{~mm}$ and a plano-convex lens with focal length of $50 \mathrm{~mm}$ arranged as an afocal telescope. In some experiments, the second lens is replaced by one with a focal length of $125 \mathrm{~mm}$ and the external cavity length is increased correspondingly.

The VCSEL has a $200 \mu \mathrm{m}$ circular aperture defined by an oxide layer providing current and optical confinement and emits through the transparent substrate ("bottom emitter," [12]) at approximately $976 \mathrm{~nm}$ at room temperature. It is grown by MBE, and the wafer has a high homogeneity on long scales with a variation of the cavity resonance by less than $0.15 \%$ over the central $40 \mathrm{~mm}$.

A volume Bragg grating (VBG) operated at normal incidence is used as a frequency-selective element. It has a single reflection peak at $981.1 \mathrm{~nm}$ and a reflection bandwidth of $0.2 \mathrm{~nm}$ FWHM with a maximal reflectivity $R \approx 0.99$. We operate the VCSEL at $80{ }^{\circ} \mathrm{C}$ in order to temperature tune the emission close to $981 \mathrm{~nm}$ such that the VCSEL wavelength approaches the reflection peak of the VBG. At this temperature, the VCSEL threshold is 
effectively infinite, and the device only lases due to feedback.

The light is coupled out of the cavity via the Fresnel reflection of a wedged beam sampler. The near field intensity distribution of the VCSEL is then imaged onto a charge-coupled device (CCD) camera. In another arm, the far field intensity distributed is monitored.

Figure 1(a) shows the spontaneous emission emitted below threshold. It is essentially featureless, indicating that the gain is quite homogeneous. The enhancement at the perimeter is due to current crowding at the oxide aperture [12]. As a consequence, the free-running laser is only operating at the perimeter, and the emission is also strongest at the perimeter-and starts there at threshold-for the case of feedback by a plane mirror, i.e., without frequency-selection (Fig. 1(i)). The latter shows some structures within the aperture, but with the exception of two "defect" lines, they are not very pronounced.

This is very different for the case of feedback by the VBG (Figs. 1(b)-(h)) where the laser starts to emit abruptly at very distinct spots (Fig. 1(b)) called solitons $[\underline{8}, 10]$. Increasing the current, the emission spreads over the aperture. Solitons arise at new positions (Figs. 1(c)(f)), and solitons formed previously give way to lower amplitude and more extended states, which correspond to off-axis emission. In other regions, the lasing intensity starts to grow in a continues way, taking about 8-10 mA to reach the $90 \%$ point of emission [13]. These observations can be interpreted in the following way: Initially, the cavity resonance of the VCSEL is at a higher frequency ("blue"-shifted) than the reflection peak of the VBG. Ohmic dissipation due to the current increase heats the VCSEL and causes the resonance to red-shift. The first locations coming into resonance with the VBG are the most "reddish" ones of the disorder induced cavity resonance fluctuations. Lasing sets in locally, and the solitons form due to nonlinear beam shaping. If the current is increased further, the cavity resonance at these reddish location overshoots the reflection peak of the grating,
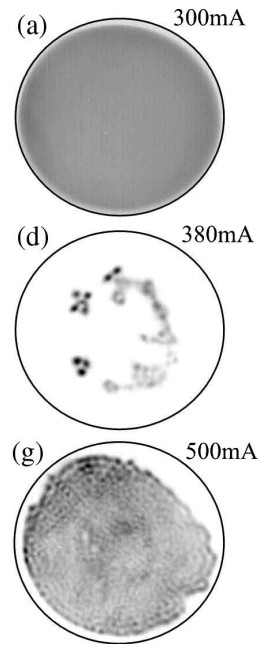
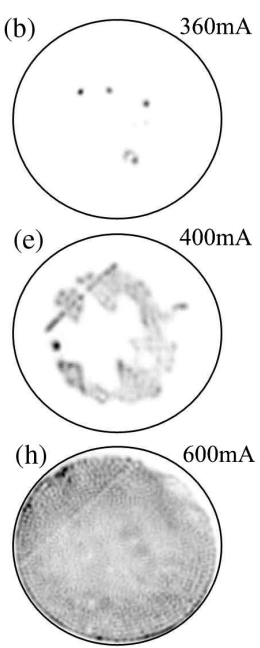
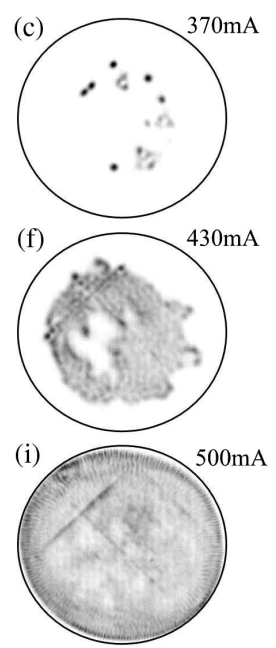

Fig. 1. Examples for near field intensity observed for increasing current. (a)-(h) Feedback with VBG, (i) feedback with plane mirror. The intensity is depicted in a linear gray scale with black denoting high intensity. The subpanels (b)-(h) are scaled to the maximum intensity occurring in the whole sequence. and on-axis states are no longer in resonance, but off-axis high-order modes are. In addition, the more "bluish" parts of the cavity come into resonance and start to emit. Hence the emission is spreading over the aperture.

These observations motivate the idea to use the difference in threshold at different locations to create a detuning map by using the tuning rate of the cavity, which can be measured for cavity modes without frequency-selective feedback, to convert from current to frequency. Initially, the VCSEL is biased just below the threshold for the first soliton. The current is then increased in $1 \mathrm{~mA}$ increments, and at each current, the near field intensity distribution is recorded. The images are converted from gray scale to a binary black and white image with a threshold in between the spontaneous emission level and the lasing states $\left(1 / e^{2}\right.$ point of intensity of the maximal amplitude). All of these binary images are then added together to produce one image in which the parts of the cavity that begin to lase first have the highest value, and vice-versa. This is then normalized to a detuning using the known cavity tuning rate of $0.0035 \mathrm{~nm} / \mathrm{mA}$.

The result is shown in Fig. 2(a). Obviously, there is a lot of small-scale disorder within the $200 \mu \mathrm{m}$ aperture on top of what seems to be a radial gradient. The most dark red locations are those where emission starts first. Typical length scales of disorder are about $10 \mu \mathrm{m}$ and they span some tens of GHz. The distinct "defect" line in the upper left part of the aperture has a width of $8 \mu \mathrm{m}$ a)

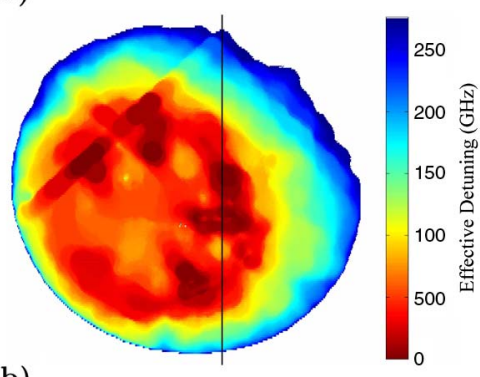

b)

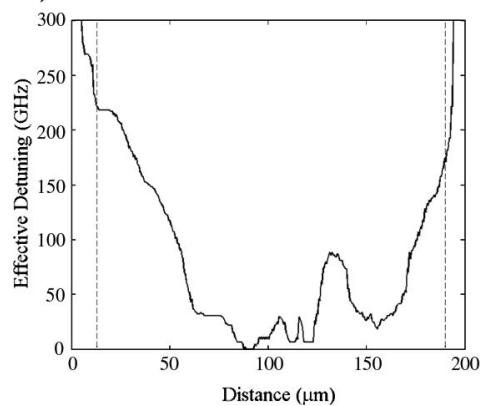

c)

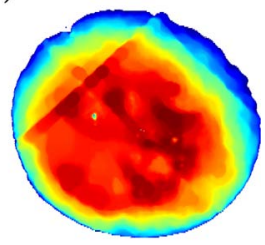

d)

e)

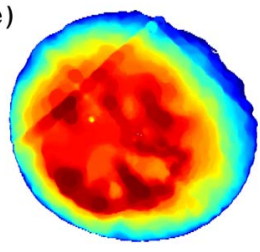

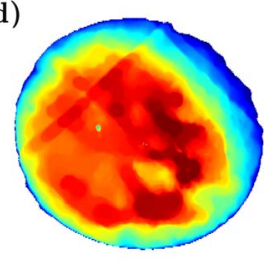

Fig. 2. (Color online) Disorder map of VCSEL as explained in the text. The pseudocolors denote the local detuning of the cavity resonance with respect to the location with the lowest frequency. (a) Short cavity; (b) cut through the two-dimensional (2D) map in (a) along the black vertical line; (c) long cavity; (d), (e) long cavity, VBG rotated by $\pm 90^{\circ}$ compared to (c). The dashed lines in (c) denote the radial distances where the emission without frequency-selective feedback is, i.e., in images as in Fig. 1 subpanels (a) and (i) peaks. Outside this range, the notion of an "effective detuning" is not valid because the emission is strongly affected by the drop in gain and not by the properties of the cavity resonance. 
and a depth of $24 \mathrm{GHz}$. A cut through the map is shown in Fig. 2(b), which illustrates very well the small scale irregularities and the depth of the resulting potential wells and ridges.

The length scales observed are similar to the ones obtained in the injected VCSEL amplifier [9], but there it is difficult to assess the depth of fluctuations, as discussed in the introduction. We do not have a direct comparison with reflection measurements for our device, because our tunable laser is not of sufficient quality, but the numbers are in agreement with values in the literature $[5,11]$. Reference [5] reports a variation of a few hundred $\mu \mathrm{eV}$ $(300 \mu \mathrm{eV} \approx 70 \mathrm{GHz})$ and length scales of $5-20 \mu \mathrm{m}$. A simple calculation shows that already a monolayer variation of $\Delta L \approx 0.3 \mathrm{~nm}$ corresponds to a frequency variation of $\Delta \nu \approx 76 \mathrm{GHz}$. Since the cavity linewidth of a low-loss VCSEL is about $0.1 \mathrm{~nm}$ or $30 \mathrm{GHz}$, this explains the extreme sensitivity of the feedback patterns to disorder.

The measurements obtained are robust as evidenced, for example, by the images in Figs. 2(c)-(e), which are obtained in a different external cavity configuration (the longer cavity mentioned in the setup section), though there are small quantitative deviations between the maps in Figs. 2(a)-(e)). Between the subpanels in Figs. 2(c)-(e), the VBG was rotated by $90^{\circ}$, again leaving the map essentially the same. These checks demonstrate that we probe disorder in the VCSEL and not in the VBG. The nonreproducible fine details are mainly related to minute changes of the alignment of the VBG. Similar sensitivity to alignment and wavefront distortion will occur if probing with a tunable laser.

The lower limit of spatial resolution is given by the minimal area in a plano-planar resonator coupled by diffraction and can be estimated to be about $4 \mu \mathrm{m}$ for our device. This is in good agreement with the minimum length scales appearing in the subpanels of Fig. 2. It should be noted that - due to the disorder-the nonsolitonic "extended" states are also changing on comparable, i.e., small, length scales (Figs. 1(c)-(e); see also [14]). The spectral resolution is given by the accuracy and reproducibility of the threshold determination. Since, in the case of a continuous switch-on, the transition between the spontaneous emission background to $90 \%$ of the final amplitude takes place in a current range of about 8$10 \mathrm{~mA}$, we estimate the resolution to be better than $0.03 \mathrm{~nm}$ or $10 \mathrm{GHz}$. The nonlinear frequency shift at the abrupt switch-on of a soliton is in the same range; the variation of emission wavelength in the initial soliton region is about $0.06 \mathrm{~nm}$ for the short cavity and $0.03 \mathrm{~nm}$ for the long cavity [13]. This might lead to a slight over- estimation of the depth of the "wells" in which the solitons are nucleating by up to $0.03 \mathrm{~nm}$ or $10 \mathrm{GHz}$.

It is important to mention that the detuning obtained by this method (as well as in [9]) is actually an "effective" detuning, i.e., temperature and carrier variations affecting the refractive index and thus the cavity resonance in addition to the growth fluctuations. The temperature is highest in the center, whereas the carrier density is highest at the perimeter. Both variations lead to a reduction of refractive index at the perimeter compared to the center and thus to an increase of resonance frequency at the boundary, as observed in Fig. 2. This can be estimated to lead to a detuning variation of the order of $\Delta \nu \approx 60-$ $190 \mathrm{GHz}$ compatible with observation (see the region in Fig. 2(b) within the dashed lines).

N. Radwell and Y. Noblet gratefully acknowledge support from an EPSRC DTG. We are grateful to W. J. Firth and G.-L. Oppo for useful discussions.

\section{References}

1. H. Q. Hou, H. C. Chui, K. D. Choquette, B. E. Hammons, W. G. Breiland, and K. M. Geib, IEEE Photon. Technol. Lett. 8, 1285 (1996).

2. M. Gurioli, F. Bogani, L. Cavigli, H. M. Gibbs, G. Khitrova, and D. S. Wiersma, Phys. Rev. Lett. 94, 183901 (2005).

3. H. Pier and E. Kapon, Opt. Lett. 22, 546 (1997).

4. S. Hegarty, G. Huyet, J. G. McInerney, and K. D. Choquette, Phys. Rev. Lett. 82, 1434 (1999).

5. D. Sanvitto, D. N. Krizhanovskii, D. M. Whittaker, S. Ceccarelli, M. S. Skolnick, and J. S. Roberts, Phys. Rev. B 73, 241308(R) (2006).

6. S. Barland, J. R. Tredicce, M. Brambilla, L. A. Lugiato, S. Balle, M. Giudici, T. Maggipinto, L. Spinelli, G. Tissoni, T. Knödel, M. Miller, and R. Jäger,Nature 419, 699 (2002).

7. F. Pedaci, S. Barland, E. Caboche, P. Genevet, M. Giudici, J. R. Tredicce, T. Ackemann, A. J. Scroggie, W. J. Firth, G.-L. Oppo, G. Tissoni, and R. Jäger, Appl. Phys. Lett. 92, 011101 (2008).

8. Y. Tanguy, T. Ackemann, W. J. Firth, and R. Jäger, Phys. Rev. Lett. 100, 013907 (2008).

9. F. Pedaci, G. Tissoni, S. Barland, M. Giudici, and J. R. Tredicce, Appl. Phys. Lett. 93, 111104 (2008).

10. N. Radwell and T. Ackemann, IEEE J. Quantum Electron. 45, 1388 (2009).

11. J. L. Oudar, R. Kuszelewicz, B. G. Sfez, J. C. Michel, and R. Planel, Opt. Quantum Electron. 24, S193 (1992).

12. M. Grabherr, M. Miller, R. Jäger, R. Michalzik, U. Martin, H. J. Unold, and K. J. Ebeling, IEEE J. Sel. Top. Quantum Electron. 5, 495 (1999).

13. Y. Noblet and T. Ackemann, arXiv 1202.0418 (2012).

14. S. Barland, F. Marino, M. Giudici, J. R. Tredicce, and S. Balle, Appl. Phys. Lett. 83, 2303 (2003). 